\title{
Supporting Information for: Electrostatics Trigger Interfacial Self-Assembly of Bacterial Ice Nucleators
}

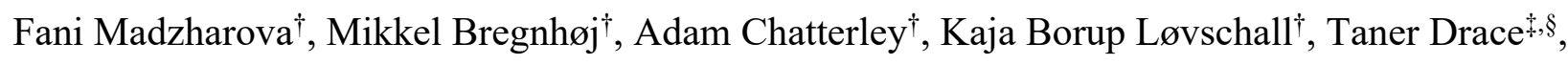
Lasse Sander Andersen Dreyer ${ }^{\ddagger}$, Thomas Boesen ${ }^{\ddagger}, 8$ and Tobias Weidner ${ }^{\dagger *}$

'Department of Chemistry, Aarhus University, Aarhus C, Denmark

Department of Molecular Biology and Genetics, Aarhus University, Aarhus C, Denmark \$Interdisciplinary Nanoscience Center - iNano, Aarhus University, Aarhus C, Denmark

E-mail: weidner@chem.au.dk

Phone: +4593508509 


\section{D IR difference spectra}
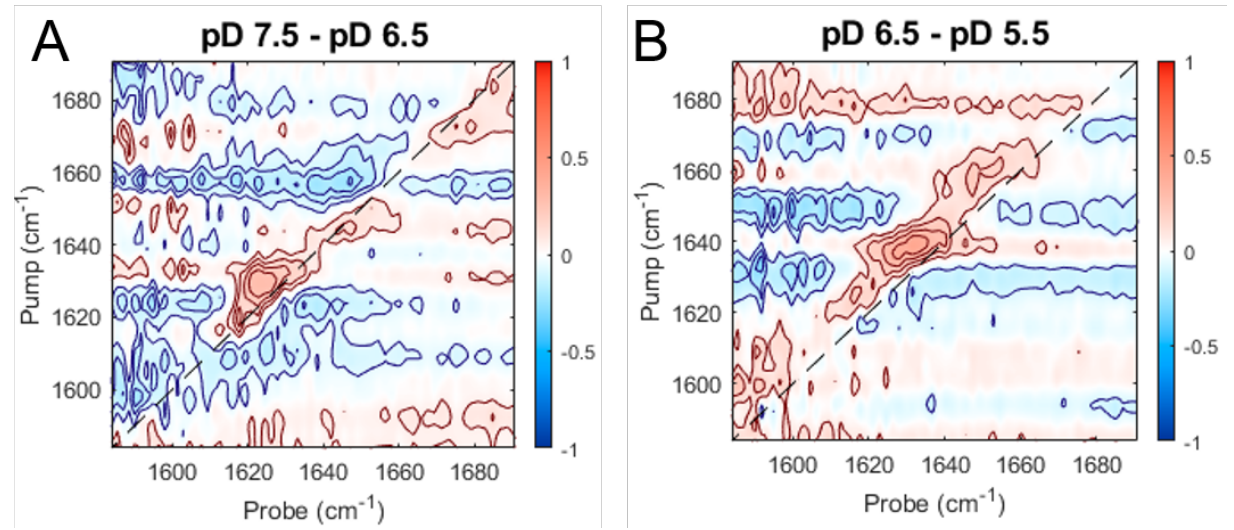

Figure S1: Difference 2D-IR spectra between (A) pD 7.5 and pD 6.5, and (B) pD 6.5 and pD 5.5. The spectra in Figure 2 from the main paper were normalized to calculate the spectra A and B here. The color bars are presenting the difference between normalized spectra in arbitrary units. 


\section{SFG fitting analysis}

SFG spectra were fitted according to:

$$
\chi_{\mathrm{eff}}^{(2)}(\omega)=\chi_{\mathrm{NR}}^{(2)}+\sum_{q} \frac{A_{q}}{\omega-\omega_{q}+i \Gamma_{q}}
$$

where $\Gamma_{\mathrm{q}}, \mathrm{A}_{\mathrm{q}}$, and $\omega_{\mathrm{q}}$ are the FWHM, amplitude, and resonant frequency of the qth vibrational mode, respectively, and $\chi_{\mathrm{NR}}^{(2)}$ and $\chi_{\mathrm{eff}}^{(2)}$ are the nonresonant background and effective second-order nonlinear susceptibility tensors, respectively. $\chi_{\mathrm{NR}}^{(2)}=A_{\mathrm{NR}} e^{i \Phi_{N R}}$, where $A_{N R}$ and $\Phi_{N R}$ are the amplitude and phase, respectively, for the nonresonant background.

Table S1: Fitting parameters for the SFG spectra in the amide region (Figure 2).

\begin{tabular}{|l|r|r|r|r|l|}
\hline sample & $A_{\text {NR }}$ & $\varphi_{\text {NR }}$ & $\omega_{\mathrm{q}}\left(\mathrm{cm}^{-1}\right)$ & $\Gamma_{\mathrm{q}}\left(\mathrm{cm}^{-1}\right)$ & $\mathrm{A}_{\mathrm{q}}$ \\
\hline pD 7.9 & 0.014 & 2.987 & 1639 & 23.9 & 3.049 \\
\hline & & & 1550 & 27.7 & 1.210 \\
\hline & & & 1516 & 27.7 & 1.239 \\
\hline & & & 1488 & 14.3 & 0.550 \\
\hline pD 7.4 & 0.023 & 1.856 & 1638 & 25.7 & 2.910 \\
\hline & & & 1546 & 32.1 & 1.329 \\
\hline & & & 1511 & 26.3 & 0.938 \\
\hline & & & 1486 & 13.5 & 0.529 \\
\hline pD 6.9 & 0.045 & 1.283 & 1638 & 27.0 & 1.267 \\
\hline & & & 1547 & 15.0 & 0.134 \\
\hline & & & 1519 & 32.8 & 0.718 \\
\hline & & & 1485 & 17.6 & 0.533 \\
\hline pD 6.4 & 0.017 & 2.009 & 1636 & 29.0 & 1.035 \\
\hline & & & 1546 & 37.1 & 0.748 \\
\hline & & & 1510 & 19.0 & 0.211 \\
\hline & & & 1489 & 11.5 & 0.178 \\
\hline pD 5.9 & 0.021 & 3.246 & 1645 & 46.3 & 1.137 \\
\hline & & & 1547 & 48.1 & 1.034 \\
\hline & & & & &
\end{tabular}


Table S2: Fitting parameters for the SFG spectra in the OD region (Figure 4).

\begin{tabular}{|c|c|c|c|c|c|}
\hline sample & $A_{N R}$ & $\varphi \mathrm{NR}$ & $\omega_{\mathrm{q}}\left(\mathrm{cm}^{-1}\right)$ & $\Gamma_{\mathrm{q}}\left(\mathrm{cm}^{-1}\right)$ & $A_{q}$ \\
\hline \multirow[t]{6}{*}{ pD 7.9} & 0.033 & 0.514 & 2360 & 27.89 & 1.392 \\
\hline & & & 2415 & 42.32 & 1.614 \\
\hline & & & 2490 & 68.81 & 3.700 \\
\hline & & & 2710 & 172.22 & 4.797 \\
\hline & & & 2860 & 4.90 & 0.109 \\
\hline & & & 2835 & 24.32 & 0.718 \\
\hline \multirow[t]{6}{*}{ pD 7.4} & 0.029 & 0.584 & 2362 & 25.41 & 0.854 \\
\hline & & & 2417 & 39.24 & 1.044 \\
\hline & & & 2494 & 72.30 & 3.632 \\
\hline & & & 2680 & 156.69 & 5.823 \\
\hline & & & 2853 & 7.66 & 0.185 \\
\hline & & & 2827 & 26.74 & 0.663 \\
\hline \multirow[t]{6}{*}{ pD 6.9} & 0.017 & 1.082 & 2365 & 16.49 & 0.299 \\
\hline & & & 2417 & 36.83 & 0.572 \\
\hline & & & 2502 & 67.12 & 2.073 \\
\hline & & & 2713 & 161.48 & 5.962 \\
\hline & & & 2836 & 26.86 & 0.743 \\
\hline & & & 2860 & 6.81 & 0.154 \\
\hline \multirow[t]{6}{*}{ pD 6.4} & 0.018 & 1.899 & 2363 & 12.64 & 0.212 \\
\hline & & & 2415 & 39.73 & 0.473 \\
\hline & & & 2495 & 50.29 & 1.046 \\
\hline & & & 2702 & 153.14 & 4.381 \\
\hline & & & 2827 & 34.25 & 0.841 \\
\hline & & & 2852 & 13.76 & 0.450 \\
\hline
\end{tabular}




\section{Surface pressure measurements}

The surface pressure was measured with a Dyne probe instrument (Kibron, Finland). For these experiments, an ice nucleating protein with the same sequence as InaZ9R but without the purification-tag at the N-terminus was used at a concentration of $0.25 \mathrm{mg} / \mathrm{mL}$ in $\mathrm{PBS}-\mathrm{D}_{2} \mathrm{O}$ and $0.012 \mathrm{M} \mathrm{DCl}$ was injected in the subphase to decrease the $\mathrm{pD}$ gradually. The surface pressure values were read out after equilibration.

Table S3: Surface pressure for the ice protein at different $\mathrm{pD}$.

\begin{tabular}{|c|c|}
\hline sample & Surface pressure $(\mathrm{mN} / \mathrm{m})$ \\
\hline pD 7.9 & 20 \\
\hline pD 7.4 & 24 \\
\hline pD 6.9 & 29 \\
\hline pD 6.4 & 31 \\
\hline pD 5.9 & 35 \\
\hline
\end{tabular}

The tag-free InaZ9R protein was expressed and purified using immobilized metal affinity chromatography as described for InaZ9R in the Materials and Methods section of the main paper. The fractions containing InaZ9R were then cleaved using His-tagged TEV protease over night, and purified using reversed immobilized metal affinity chromatography, hereby binding uncleaved InaZ9R, cleaved purification tag, and TEV protease. The flow-through containing tag-free InaZ9R was collected and concentrated using spin-concentrators. The sample was then run on size-exclusion chromatography column and treated as described for InaZ9R. 


\section{Circular dichroism (CD) measurements}

UV-CD spectra were recorded with a Jasco 810-J spectropolarimeter using a $1 \mathrm{~mm}$ path length quartz cuvettes. Tag-free InaZ9R was dissolved in PBS- $\mathrm{D}_{2} \mathrm{O}$ at a concentration of $0.2 \mathrm{mg} / \mathrm{mL}$ and $0.012 \mathrm{M} \mathrm{DCl}$ was added to adjust the $\mathrm{pD}$.

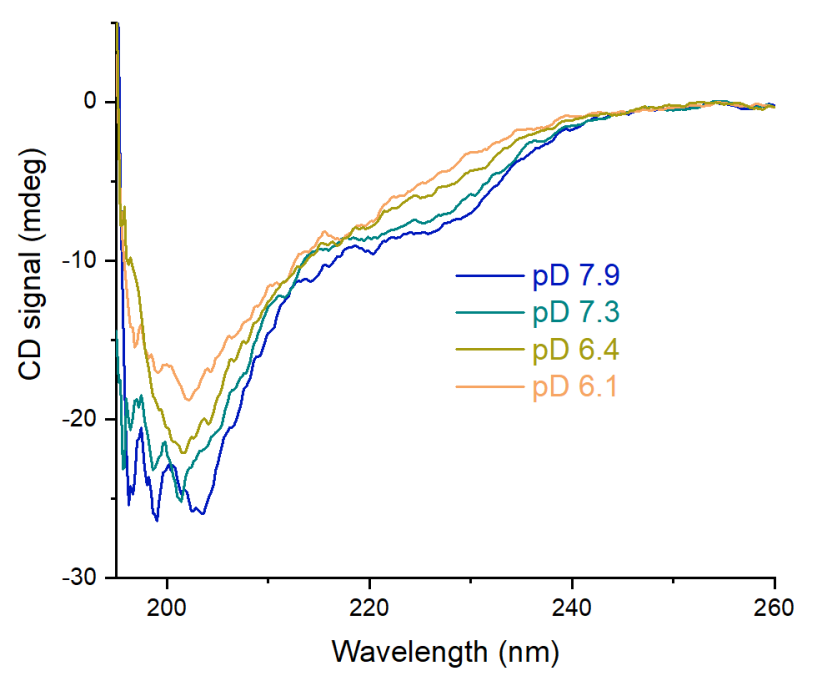

Figure S2: UV-CD spectra of the tag-free ice nucleating protein at different $\mathrm{pD}$ values.

The spectra show a strong negative contribution at $200 \mathrm{~nm}$ with a broad shoulder around $225 \mathrm{~nm}$, consistent with what we have previously recorded for InaZ9R at neutral pH (Nat. Commun. 2021, 12, 1183). The UV-CD spectra are dominated by the $\mathrm{N}$ - and C-termini structures with only slight contributions from the $\beta$-helical core structure. With decreasing $\mathrm{pD}$, the total signal slightly decreases, possibly due to protein precipitation, but the overall shape of the spectra is very similar, indicating that the protein structure within this $\mathrm{pD}$ range remains largely unaltered, in agreement with the 2D-IR data of InaZ9R. 